\title{
Novel approach of UWB Multi-band system based on orthogonal function for transports applications
}

\author{
F.Elbahhar ${ }^{1}$, A.Lamari ${ }^{2}$, A.Rivenq ${ }^{2}$, J.M.Rouvaen ${ }^{2}$, M.Heddebaut ${ }^{1}$, T.Boukour ${ }^{3}$, and L.Sakkila ${ }^{2}$ \\ 1 INRETS, LEOST, F-59666 Villeneuve d'Ascq, France \\ 2 UVHC, IEMN-DOAE, F-59313 Valenciennes, France \\ 3 E-BLINK, F-91800 Boussy-Saint-Antoine, France
}

Received: date / Revised version: date

\begin{abstract}
Ultra Wide Band (UWB) is based on the transmission of very short pulses with relatively low energy. This technology may see increased use in the wireless communications and sensing application. Having the available bandwidth of $7.5 \mathrm{GHz}$ and the minimum signal bandwidth of $500 \mathrm{MHz}$, UWB systems can be divided into two groups: Impulse communication system and Multi-band system. In multi-band, the spectrum is divided into several sub-bands. In this paper, we propose this technique for subway transport systems; especially for communication between trains or train- infrastructure. So, the main purpose of this paper is to make use of the original mathematical tools called orthogonal functions to allow a multi-user communication system for multi band UWB transmission. The BER values will be calculated and analyzed using computer simulations in the presence of multi-user interference, in order to evaluate the performances of the proposed system. These BER values will be given for two cases: the users in the same sub-band and a single user per sub-band. We will show that the proposed orthogonal waveforms give good performances in terms of BER and are able to reduce multi-user interference. The proposed method has many potentials advantages in terms of complexity and implementation.
\end{abstract}

\section{Introduction}

Ultra Wide Band (UWB) radio is a candidate technology for subway transports systems especially for communication between trains or between train-infrastructure. It uses very short pulses rather than the traditional method of modulating sinusoidal carrier [1]. The large bandwidth of an UWB system is dominated by the pulse shape and duration. This allows UWB systems to operate with a low power spectral density and implies that the UWB signal may be kept near or below the noise floor of hostile detection devices.

The proposed definition by the Federal Communication Commission (FCC) for UWB transmission: It considers as UWB any signal, which has fraction bandwidth $B_{f}$ larger than 0.25 , or which occupies a bandwidth greater than $500 \mathrm{MHz}$. The Fractional bandwidth is defined as:

$$
B_{f}=2 \frac{\left(f_{h}-f_{l}\right)}{f_{h}+f_{l}}
$$

Where $f_{h}$ and $f_{l}$ represents the highest and the lowest $-10 \mathrm{~dB}$ frequency points respectively. This definition of UWB bas been adopted by the majority of involved parties.

Several properties speak in favour of the use of UWB technique such as: high data rate, low probability of interception and detection, system simplicity, low cost, reduced average power consumption, low sensitivity to the near-far problem or immunity to interference [2][3].

UWB technologies can be divided into two groups: single band (pulsed) systems and multi-band systems using a single carrier in each sub-band. The classical multi-band UWB system consists in dividing the bandwith into several sub-bands using a traditional multi-access technique such as the frequency hopping $(\mathrm{FH})$ technique to address each sub-band [4] [5]. Another multi-band system based on orthogonal frequency division multiplexing (OFDM) techniques on each sub-band has been proposed (MB-OFDM).

During its standardisation attempted by IEEE 802.15.3a group [6], its good properties for capturing efficiently multipath energy, mitigating interference and achieving large throughput have been demonstrated. In the MB-OFDM scheme, the signals consist of 128 sub-carriers spaced by $4.125 \mathrm{MHz}$ apart and utilize the 3.1 to $10.6 \mathrm{GHz}$ range in 14 sub-bands, the bandwidth of each sub-band being set to $528 \mathrm{MHz}$. But the drawback of this type of system is that the transmitter is slightly more complex because it requires an inverse FFT (IFFT) and the peak-to-average ratio may be slightly higher than the ones of the other pulsed multi-band approach. All multi-band systems are attractive radio techniques that coexist with other narrowband signals and have interesting properties. In this study, we propose the novel approach based on the orthogonal functions called Modified Gegenbauer Functions 
(MGF) in a multi-band UWB communication,occupying the 3 to $6 \mathrm{GHz}$ frequency band, for subway transport systems.

\section{Proposed system}

The proposed system concerns the subway application, for train to train or train to infrastructure communication. Generally, the subway line is divided in parts called sections of about $500 \mathrm{~m}$ length. When a train is in a section, it is declared to be engaged and no coach can go in until the train leaves it. This is the safety system adopted in most of the current networks. In this scope, only a few trains (say from 2 to 4 , due to limited emitted power) will be allowed to receive or transmit data or video in any given area, including broadcast messages to passengers or security information sent to or from the control center, like train status or problems encountered on the way or on-board.

For this wireless communication application, a sufficiently low BER, between $10^{-4}$ and $10^{-3}$, is required and a maximum data rate of about $20 \mathrm{Mbits} / \mathrm{s}$ is sufficient. Although multi-band OFDM offers communication at data rates greatly exceeding those needed in our application, this is obtained against range of communication (a few meters in this case, against at least one hundred of meters needed in our application). Multi-band OFDM will not adapt for our system. But the principle of multi-band modulation has been retained, with only a few sub-bands.

In this paper, we propose to use the Modified Gegenbauer Functions (MGF), derived from orthogonal polynomials [2], to achieve the multiple access needed in our application.

The Modified Gegenbauer functions permit to have the multi access (CDMA) through propagation channel so replace the pseudo-random code and offer good performance in terms of Bit Error Rate (BER). The orthogonal functions [9] are introduced as basis functions for the pulse shapes in a multi-band system. The Modified Hermite or the Modified Gegenbauer functions are examples of these orthogonal waveforms that can also be used in the wireless UWB communication system [7]. It is shown in [2] that MGF offer good performance for multi-user UWB communication. The orthogonality of these MGF is then exploited here to construct a multi-band UWB communication system and to reduce the Multi-User Interference (MUI) in asynchronous communications.

This paper is organized as follows. Section 2 gives the properties of MGFs. Section 3 presents the proposed system. In section 4, the UWB system using orthogonal MGFs performances are presented in terms of BER for different SNR values in two cases: several users in the same sub band and interferences between nearby sub-bands. Finally, Section 5 summarizes the results and presents our future work.

\section{Modified Gegenbauer Functions}

The orthogonal functions have been used in many different mathematical and physical areas [7], but these functions are seldom used in communication systems where sinusoidal (modulated carrier) communication is the most common method. By definition, a family of polynomials $f_{n}(x)$ (where $\mathrm{n}$ stands for the degree of polynomial) is said to be orthogonal on the definition interval [a; b], if it satisfies the condition:

$$
\int_{a}^{b} w(x) f_{n}(x) f_{m}(x) d x=0
$$

with $n$ and $m$ unequal non negative integers and where $w(x)$ is the weight for the generalized scalar product. Correlation will be used here in the received signal detection process.Thus, in order to view the scalar product as a correlation at zero delay, the waveforms used for coding user data will be:

$$
F_{m}(x)=f_{m}(x) \frac{\sqrt{w(x)}}{\int_{a}^{b} w(x) f_{m}^{2}(x) d x}
$$

assuming that $w(x)$ is strictly positive over the definition interval. Equation (2) may then be rewritten as a standard scalar product:

$$
\int_{a}^{b} F_{n}(x) F_{m}(x) d x=0
$$

Equation (4) insures also that the energy is normalized to unity, say to $1 \mathrm{~J}$, for each polynomial order $\mathrm{m}$ (equal width waveforms will then carry the same power). The functions $F_{n}$ constitute then an orthonormal basis for a space of functions over the definition interval [a, b].

Moreover, if the definition interval is infinite, it must be restricted to a finite one by truncation. This truncation must not be too severe: the fraction of the waveform energy which is so suppressed must remain negligible (say lower than 1

A huge number of polynomial families may be considered as, for example, the classical Hermite [7], Laguerre, Bessel and Gegenbauer polynomials. In our preceding study [2], we have shown that modified Gegenbauer functions are more adapted for the communication UWB multi users.

The modified Gegenbauer functions [2][4], permit to modulate the data and, simultaneously, allow multi-user communication. The Gegenbauer polynomials $G_{n}(\beta, x)$ use the weight function $w(x)=\left(1-x^{2}\right)^{\beta-1 / 2}$ where $\beta>-\frac{1}{2}$ is a shape parameter, $n$ is the degree of the polynomial and $x$ is the variable. These functions are orthogonal on the interval $[-1,1]$ for $m \neq n$ :

$$
\int_{-1}^{1} w(x) G_{n}(\beta, x) G_{m}(\beta, x) d x=0
$$




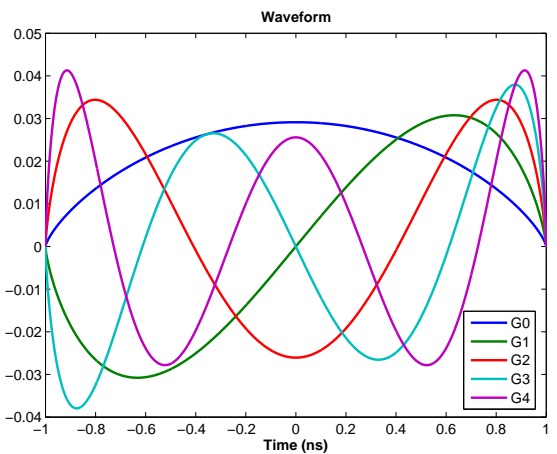

Fig. 1. Time representation for modified Gegenbauer functions of orders $\mathrm{n}=0$ to 3 .

These polynomials can be defined by the recurrence relation. Furthermore, they satisfy the differential equation.

$G_{n}(\beta, x)=2 \frac{n+\beta-1}{n} x \cdot G_{n-1}(\beta, x)-\frac{n+2 \beta-2}{n} G_{n-2}(\beta, x)$

Their expressions for the first few orders are given by the following equation:

$$
\begin{aligned}
& G_{0}(\beta, x)=1 \\
& G_{1}(\beta, x)=2 \beta x \\
& G_{2}(\beta, x)=\beta\left[-1+2(1+\beta) x^{2}\right] \\
& G_{3}(\beta, x)=\beta(1+\beta)\left[-2 x+(2+\beta) \frac{4 x^{3}}{3}\right] \\
& G_{4}(\beta, x)=\beta(1+\beta)\left[\frac{1}{2}-2(2+\beta) x^{2}+(2+\beta)(3+\beta) \frac{2 x^{4}}{3}\right]
\end{aligned}
$$

The Gegenbauer polynomials may be used in an UWB communication system to construct pulses with narrow widths. For this purpose, they are multiplied by the square root of the weight function $w(x)$. Our proposed modified Gegenbauer functions MGF [2] are thus given by:

$$
\Omega_{\mathrm{n}}(\beta, x)=\sqrt{w(x)} G_{n}(\beta, x)
$$

They are directly orthogonal from equation 7 :

$$
\int_{-1}^{+1} \Omega_{\mathrm{m}}(\beta, x) \Omega_{\mathrm{n}}(\beta, x) d x=\delta(m-n)
$$

The waveforms of the MGF $\Omega_{\mathrm{n}}$ are given in figure 1 , for $n=0$ to 4 and $\beta=1$. They are normalized in order to have an uniform energy of unity.

The cross-correlations between the MGF waveforms appear to be even when the pulse orders have different parities and their maximum magnitudes become smaller, as the difference between their orders increases, illustrated in figures 2 and 3.

Figure 4 shows the power spectrum densities (PSD) for the first MGF orders. The dashed lines represent again

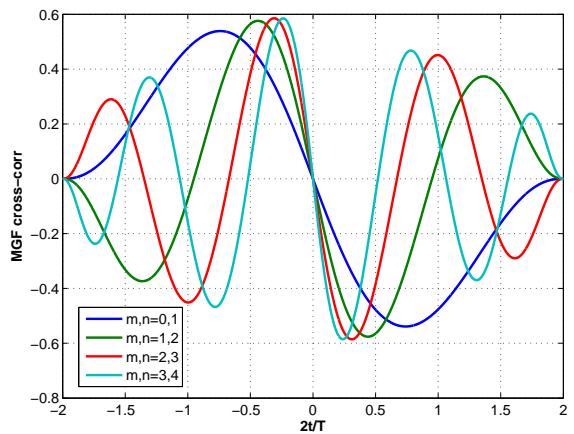

Fig. 2. Time domain representation of the cross-correlation of MGF pulses consecutive orders $\beta=1$.

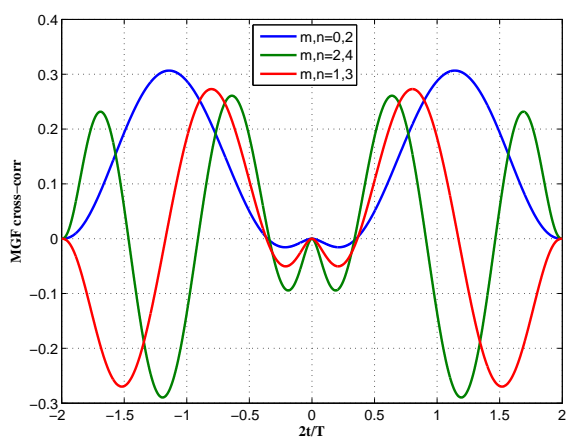

Fig. 3. Time domain representation of the cross-correlation of MGF pulses non consecutive orders $\beta=1$.
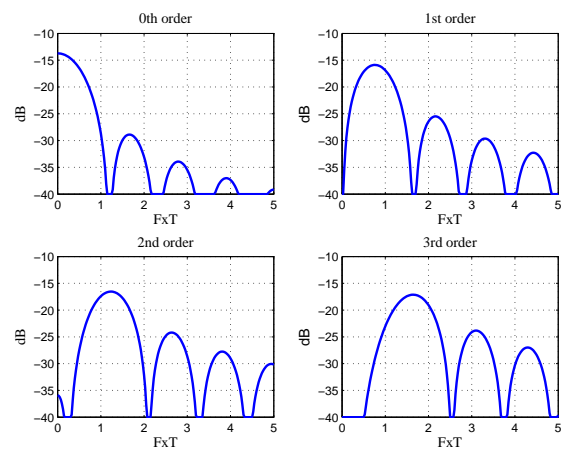

Fig. 4. PSD of Modified Gegenbauer functions.

the power level 10dB down from the maximum PSD and $\mathrm{T}$ stands for the Gegenbauer pulse duration.

\section{The proposed coded multi-bands communication system}

In our application, the frequency range from 3 to $6 \mathrm{GHz}$ will be used. In fact, this spectral area is authorized by both FCC and ETSI (European Telecommunication Standards Institute) [8] for wireless UWB applications. In order to support a maximum of 4 users and to cope with a smaller number of users, 2 sub-bands may be used for 
communication, with 0,1 or 2 user(s) sharing each given sub-band. This implies the use of two different MGF codes in the system.

One may choose, for example, to put these two communication sub- bands in the spectrum region higher than $4 \mathrm{GHz}$. To get a maximum data flow rate of $25 \mathrm{Mbits} / \mathrm{s}$, one MGF must be transmitted each 40ns, so that the MGF duration $\mathrm{T}$ (taken equal to half the symbol period Ts) will be around 20ns. Each user may be represented by a doublet $(\mathrm{m}, \mathrm{p})$, where $\mathrm{m}$ is the order of the MGF attributed to him and $\mathrm{p}=1$ or 2 , in increasing frequency order, represents the sub-band it uses. The most logical MGF order and sub-band affectation strategy, taking maximum system load (4 users) into account is as follows:

one MGF code (say $G_{n}$ is reserved to trains (from 0 to 2) going in one direction, the other code $\left(G_{n}\right)$ being attributed to trains (also from 0 to 2 ) going in the opposite direction. The two sub-bands are alternatively assigned to consecutive trains on the same side.

Several variants of the system may then be defined and studied: two different MGF codes are to be chosen among $G_{0}$ to $G_{4}$ (for which the generators are considered as realizable) and the frequency separation between the two sub-bands $\Delta F$ may be varied. For this latter purpose, a dimensionless parameter $M=\Delta F \times T$ may be introduced. Our purpose here is to characterize the MUI effects between only two users. This study allows to distinguish between two different problems. Firstly, the interferences between users sharing the same sub-band are considered. Second, the problem of interferences between users in nearby sub-bands is also addressed.

\subsection{Several users in the same sub band}

In the same sub band case, the problem is bound to the possible co-existence of several MGFs, all with different orders, in the same sub-band. The same sub-band is then shared by several users, as theoretically enabled by MGF orthogonality. Figure 5 and Figure 6 correspond to the cases $|m-n|=1$ and $|m-n|=2$, respectively. The obtained results show that the latter case is more interesting in terms of BER as expected. For example, at $\mathrm{SNR}=10 \mathrm{~dB}$, the BER is around $5 \cdot 10^{-4}$ in the first case and around $7.10^{-5}$ in the second one. Moreover, the BER depends only weakly of the specific values of $m$ and $n$, but is rather governed by their difference.

Satisfactory solutions for low BER (lower than $10^{-4}$ ) communication are found for each $(\mathrm{m}, \mathrm{n})$ pair with $m \neq n$. Moreover, the BER is a decreasing function of the difference between the two orders $|m-n|$.

There will be at most two users in each sub-band in our proposed system. In this case, all $(\mathrm{m}, \mathrm{n})$ pairs are possible candidates for their orders, but the BER decreases more and more as the difference in these orders, $|m-n|$, increases.

Thus, the solution $|m-n|=2$ has been preferred for our system: it gives better performance than the $|m-n|=$ 1 solution and more design flexibility ( 3 alternatives) than

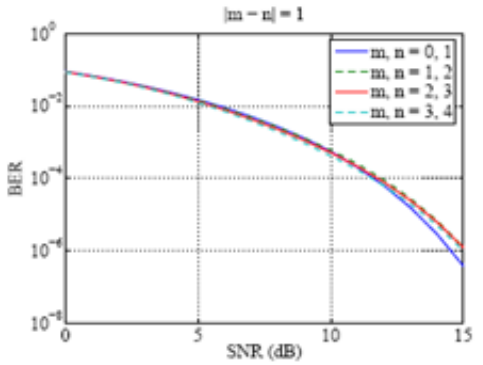

Fig. 5. Two users in the same sub-band $|m-n|=1$.

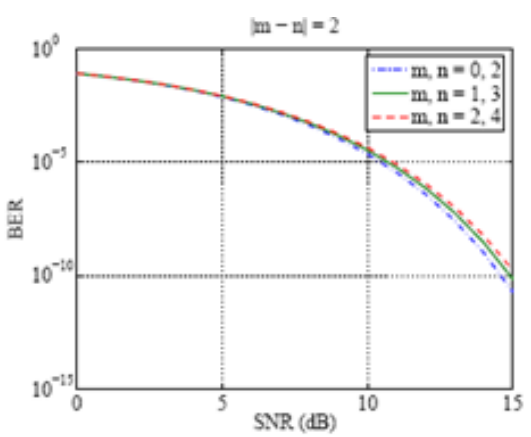

Fig. 6. Two users in the same sub-band $|m-n|=2$.

the more performing $|m-n|=3$ (2 alternatives) and $|m-n|=4$ (a single alternative) solutions.

\subsection{Interferences between nearby sub-bands}

The mutual interference of MGFs transmitted in different sub-bands will be studied here. The key parameter (apart of the involved MGF orders) is the product $M=\Delta F \times T$ of the MGF time duration $\mathrm{T}$ and the absolute value of the frequency spacing between the two sub-bands $\Delta F$. Let us assume that the two nearby sub-bands are each occupied by a single-user (MGF).

The users are assumed to be received with equal power and use the order pairs. The two users may use the same $\operatorname{MGF}(\mathrm{m}=\mathrm{n})$ or the pair of MGFs $|m-n|=2$. Moreover, the MGF waveforms are used to modulate oscillators which deliver the carriers at sub-bands centre frequencies in the multi-band system. It is not possible to maintain the phase relationship between these oscillators and a random phase difference term $\phi_{i j}$ appears between user i and $\mathrm{j}$.

The cross-correlation term can be given by the equation (10):

$R_{i j}\left(\tau_{i j}, \phi_{i j}, \Delta f_{i j}\right)=\int_{-T / 2}^{T / 2} G_{m}(t) G_{n}\left(t-\tau_{i j}\right) \cos \left(2 \pi \Delta f_{i j}+\phi_{i j}\right) d t$.

The BER has been computed for different SNR values and for $\beta=1$. We can conclude that the orders of the 


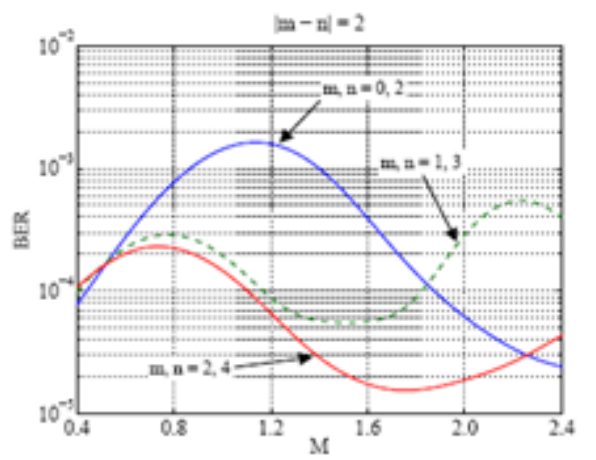

Fig. 7. Two users in the same sub-band.

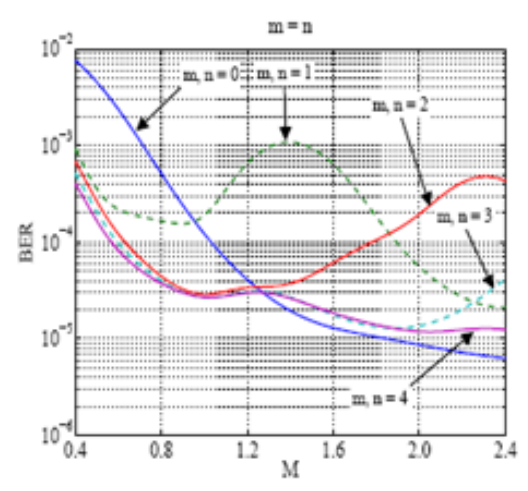

Fig. 8. Single user per sub-band $m=n,|m-n|=2$.

2 MGFs co-existing in each sub-band are chosen of the same parity, say $(0,2),(1,3)$ or $(2,4)$. It is desired to preserve spectral efficiency: the frequency spacing between sub-bands (and thus parameter M) must be as low as possible (without scarifying performance). This implies a full frequency range for the communication function which will not exceed $400 \mathrm{MHz}$, spanning for example from 4.6 $\mathrm{GHz}$ to $5.0 \mathrm{GHz}$.

Figures 7 and 8 give the results obtained for, respectively, $|m-n|=2$ and $m=n$, assuming $\beta=1$ and $S N R=10 d B$. A number of configurations enabling communication at low BER are observed. The curves have in fact an oscillatory character, as expected. However, the minima are not very close the optimal BER value.

The cross-correlation nullifies only for some particular sets of parameters $\tau_{i j}$ and $\phi_{i j}$ over small intervals. These intervals being larger for the $|m-n|=2$ pairs than for $\mathrm{m}=\mathrm{n} \neq 0$ ones, the BER is a little lower in the former case. The low BER for the special case $m=n=0$ comes from the unipodal nature of $G_{0}(t)$, with a resulting auto-correlation without sidelobes and a main lobe much larger than in the $m=n>0$ cases. This wider main lobe attributes a paramount effect to the cosine term in the integral of expression (10)), giving small cross-correlation (in fact auto-correlation here, since $\mathrm{m}=\mathrm{n}$ ) values.

The asymptotic behaviour thought above for the BER curves for all $(m, n)$ pairs when increasing the value of parameter $\mathrm{M}$ is well observed in Figures 9 and 10 for,

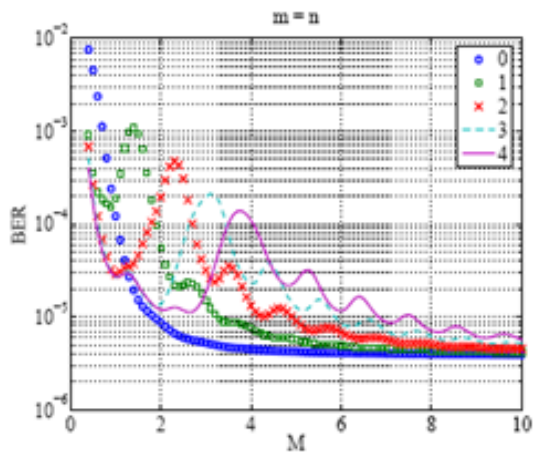

Fig. 9. Asymptotic BER limit $m=n$.

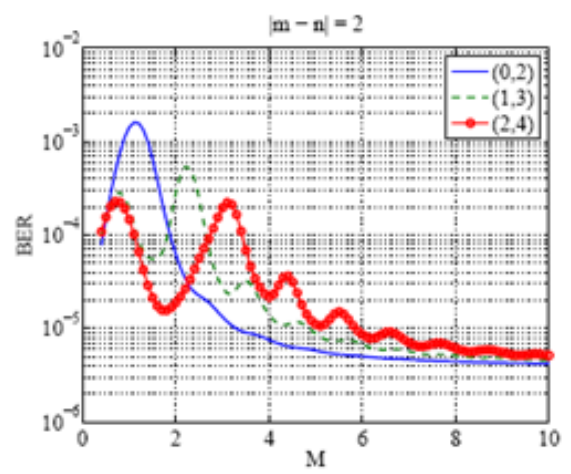

Fig. 10. Asymptotic BER limit $|m-n|=2$.

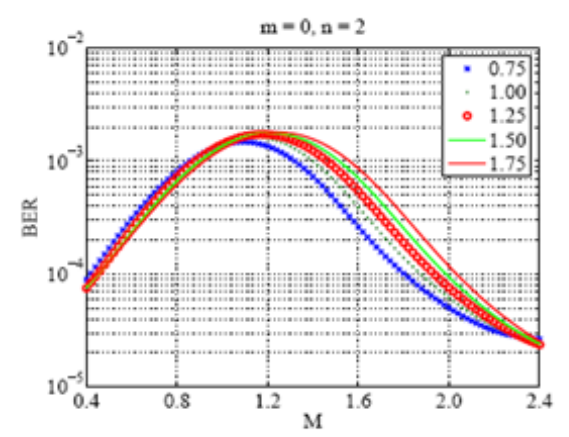

Fig. 11. Single user per sub-band - Influence of parameter $\beta(0.75$ to 1.75$)$ and $(m, n)=(0,2)$.

respectively, $\mathrm{m}=\mathrm{n}$ and $|m-n|=2$, assuming again $\beta=1$. It may then be concluded that satisfactory solutions for low BER communication exist finally for each $(\mathrm{m}, \mathrm{n})$ pair. The ranges of $\mathrm{M}$ values (for $0.4<M<2.4$ ) leading to a BER lower than $10^{-4}$ are summarized in table 1 , for the cases $m=n$ and $|m-n|=2$.

The influence of parameter $\beta$ has again been studied. As illustrated in Figure 11, it is found that, for $M$ values higher than 1.4, the main effect is a slight shifting of the BER curves. 
Table 1. Available ranges of $\mathrm{M}$ values $-\mathrm{SNR}=10 \mathrm{~dB}$

\begin{tabular}{ll}
\hline Orders $(m, n)$ & Ranges \\
\hline$(0,0)$ & {$[1.04,2.40]$} \\
$(1,1)$ & {$[1.90,2.40]$} \\
$(2,2)$ & {$[0.64,1.76]$} \\
$(3,3)$ & {$[0.60,2.40]$} \\
$(4,4)$ & {$[0.58,2.40]$} \\
\hline$(0,2)$ & {$[1.88,2.40]$} \\
$(1,3)$ & {$[1.18,1.82]$} \\
$(2,4)$ & {$[1.12,2.40]$} \\
\hline
\end{tabular}

\section{Conclusion}

In this paper, wireless UWB communication for subway transports application is proposed especially for train to train communication. So, a multi-band UWB multiple access system, using MGF pulses in an AWGN channel has been studied. The presented results show that the MGFUWB asynchronous system gives good performance in terms of BER, with reduced MUI, particularly in the most common case of 3 communicating trains, like in the full load case. The proposed system has many potentials advantages in terms of complexity and implementation. A few complementary studies will be conducted in the near future to increase the interest and improve performance of this system.

However, other advantage of the UWB technique is the possibility to have in the same architecture communication and the localization trains. In fact, positioning in difficult environments, especially indoor, represents a current limitation for localisation systems (GPS/Galileo). So, it is important to provide an indoor positioning function with a good resolution of about one centimetre. So, in order to reach this performance level, the UWB signal can be used.

In our future work the localisation system based on UWB technology for the subway transport application will be study particularly, its interactions with the communication one. The BER constraints for location are less stringent than for communication system since several consecutive pulses may be used in the process, which is more apparent to a detection problem. So the probabilities of true detection and false alarm would be more significant in this case. In addition, it has been said that the loca- tion application will use other sub-bands, lying in a widely separated part of the frequency spectrum. The problem is thus to choose, for location, a frequency separation and MGF codes, so that the impact on the BER for communication will not be significant.

\section{Acknowledgement}

We thank the French National Research Agency ANR and Transports Terrestres Promotion Northern France (ITrans), PRIMA-CARE project, which has supported this work.

\section{References}

1. Benedetto, M.-G. and B.R. Vojcic, "Ultra Wide Band (UWB) Wireless Communications: A Tutorial" Journal of Communication and Networks, Special Issue on UltraWideband Communications 5, (2003) 290-302.

2. F. Elbahhar, A. Rivenq-Menhaj and J.M Rouvaen, "Multiuser Ultra Wide Band communication system based on modified Gegenbauer and Hermite functions" Wireless Personal Communications Kluwer 34, (2005), 255-277.

3. A. Batra, J. Balakrishnan, A. Dabak and al., "TI Physical Layer Proposal for IEEE 802.15 Task Group 3a" IEEE P802.15-03/142r2-TG30, (March 2003).

4. F. Elbahhar, A. Rivenq-Menha j, J.M. Rouvaen, M. Heddebaut and T. Boukour, "Comparison between DS-CDMA and Modified Gegenbauer Functions for a multi-user communication Ultra Wide Band system" I.E.E. Proceedings Communications 152, (2005), 1021-1028.

5. R. A. Scholtz, "Mutiple Access with Time Hopping Impulse Modulation" MILCOM Boston, (1993), 447-450.

6. Vojcic, B. and Pickholtz and R.L., "Direct-Sequence Code Division Multiple Access for Ultra-Wide Bandwidth Impulse Radio," Proc. MILCOM2003 Boston (USA), (October 2003), 898-902.

7. Mohammad Ghavami, Lachlan B. Michael, Shinichiro Haruyama, Ryuji Kohno "A Novel UWB Pulse Shape Modulation System," Kluwer International Journal on Wireless Personal Communications, (2002), 105-120

8. Federal Communications Commission, Notice of Proposed Rule Making, FCC 00163, Washington, (2000) Docket 98153. 9. M Abramovitz and I.A. Stegun, Orthogonal polynomials, Handbook of mathematical functions with formulas, graphs and mathematical tables (ed.Dover, New York 1972).

10. Author, Book title (Publisher, place year) page numbers J.G. Proakis, Digital communications (ed. Mc-Graw Hill, New York 2001).

11. ETSI, "Electromagnetic compatibility and Radio spectrum Matters (ERM); Short Range Devices (SRD) using Ultra Wide Band (UWB) technology for communication purposes in the frequency range $3.1 \mathrm{GHz}$ to $10.6 \mathrm{GHz}$; Har(overview paper)" Proceedings of SPIE 28, (1991), 76-87. 